الفلى الجيفي فأجيل الثاني والثالث والرالع لتهجينينمن الهظة الهشنة

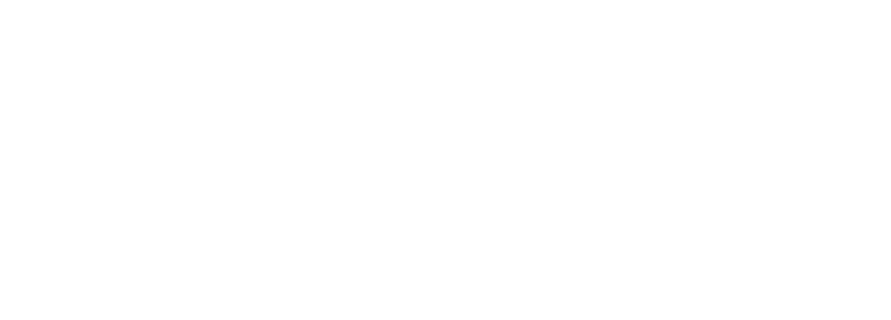

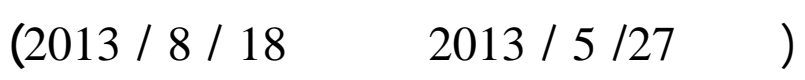

\title{
المالخص
}

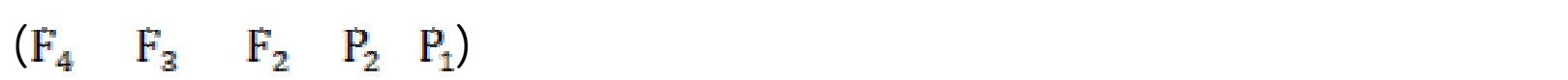

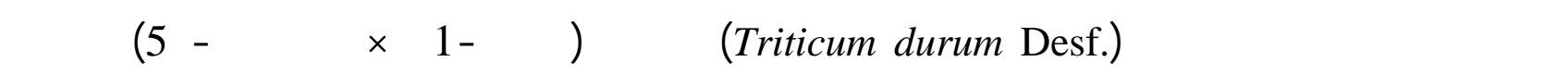
(ليدز× ولحة)، لقدير أنواع الفعل الجيني لكل من ارتفاع النبت ونسبة الكلوروفل الكلي في ورقة العلم ووقتطرد للسنالب وطول للسنبلة ومسلحة ورقة العلم وعدد عروق ورقة العلم، لستخدم أنمونجان في تحليل مكونت متوسطلت الأجيل، أنموذج المعالم الوراثية الثلاثة وأنموذج المعالم الوراثية الخصة، أوضهت نتائج

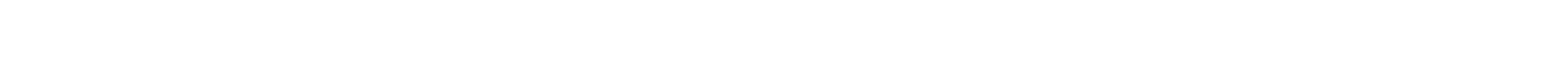
التأثيرات الإضافية وللسياية والقوقية للجينات المتعدة دورا معنويا في التعبير عن الصفلت المذكورة. الكاملت الدالة: الفعل الجيني، الحظة الخشنة، ورقة العلم، لجيل ذاتية الإخصل.

\section{Gene Action in Second, Third and Fourth Generations for Two} Durum Wheat Crosses

\author{
Najeeb K. Yousif \\ Sahir M. Aboodi \\ Department of Biology \\ College of Science \\ University of Mosul
}

\begin{abstract}
Five self-fertilized generation means $\left(P_{1}, P_{2}, F_{2}, F_{3}\right.$ and $\left.F_{4}\right)$ of two crosses in durum wheat (Triticum durum Desf.), the first (Azeghar-1 $\times$ Um-Rabie-5), and the second (Leeds $\times$ Waha), were genetically analyzed to estimate gene action for the traits: plant height, total chlorophyll percent in flag leaf, heading time, spike length, number of flag leaf venation and flag leaf area. Two models were used for analyzing the components of the generation means, parameters model and five- parameters model. The results showed that the three - parameter model was inadequate for the inheritance of all the studied traits in the
\end{abstract}




$$
\text { نجيب قاقوس يوسف وساهر متي عبودي }
$$

two crosses. Additive, dominance and epistatic effects for polygenes revealed significant role to express the studied traits.

Keywords: Gene action, durum wheat, flag leaf, self-fertilized generations.

\section{الفقمة}

بدأ الاهتملم الفعلي بعد التعرف على أعمل منل علم 1900 بأعداد برلمج وراثية للتعرف على طبيعة الفعل الجيني المسيطر على الصفلت الكمية لمحاصل الحبوب ومنها الحظة للإفاة في اعتماد برلمج تربية كفوهة ومنلسبة للانتخلب في الأجيل الانعزالية (الثاني والثالث والرابع) ويمكن التوصل إلى تلك المعلوملت بتحليل متوطلت الأجيل، إذ توفر المعلوملت الضرورية عن الأهمية النسبية للتأثيرات الإضفية وللسياسية والقوقية للجينات المتعددة وتكثف عن وجود القوق من عمه بلستخدلم لختبار مرعع كاي. وعلى الرغم من بسلة هذا التحليل لكنه ضروري لنقدير التأثيرات الجينية التي تعين الصفلت بموجب أنمونجي المعالم الوراثية الثلاثة والخمة، لذلك وضعت عة نماذج وراثية لتقدر المعالم الوراثية من المتوسطلت

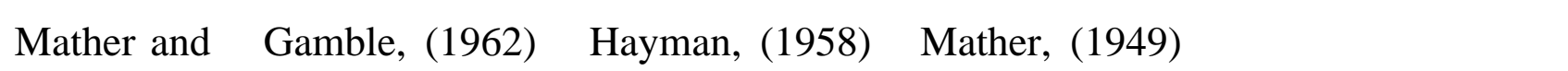
و (Jinkes, (1982) الاعتماد على الانتخلب المبلثر Direct selection عنما يكون الفعل الجيني الإضافي من المكونت الرئيسية في تعيين الصفة الكمية في حين قيترح لستخدلم برنلمج التهجين عنما يكون الفعل الجيني غير الإضافي من المكونات الرئيسية، ويستخدم الانتخلب المتكرر Recurrent selection عنما يكون الفعل الجيني الإضفي وغير الإضافي ذا تأثيرات معنوية على الصفة المدروسة. تهوف الدرلسة إلى التحليل الوراثي لمتوطلت خطسة لجيل ( F في تهجينين من الحطة الخشنة لقدير المعام الوراثية الخطة: قيمة متوطط الأبوين والتأثير الإضفي والتأثير للسيادي والتأثير الفوقي (الإضفي × الإضفين) و (للسيادي × للسيادي) للجينت المتعدة للصفلت: وقتطرد للسنال وارتقاع النبت ونسبة الكلوروفيل الكلي في ورقة العلم وطول للسنبلة وعدد عروق ورقة العلم ومسلحة ورقة العلم.

\section{المواد طرلئ الهل}

لستخدت في هذ الدرلسة خمسة لجيل لتهجينين من الحظة الخشنة (Triticum durum Desf)، التهجين الأول بين الصفين Azeghar-1 و Um- Rabie-5 والثاني بين الصقين Weeds و Waha، وقدبين بينت الحمداني (2005) نسب هذه الأصنف وكيفية لججراء التهجينت والحصول على حبوب الجيل الأول وقم الحصول على حبوب الجل الثاني F⿸ من الإخصب الذاتي لنباتلت الجيل الأول وعلى حبوب الجل 


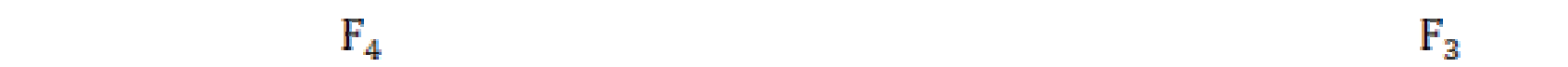

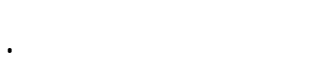

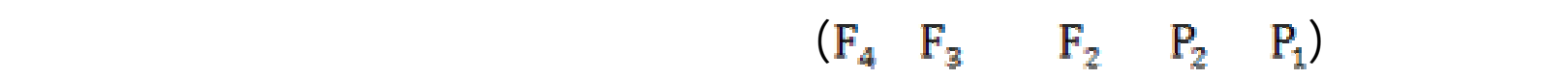
النطري (Vitavax) في حقل المحاصيل الدقلية التايع لكلية الزراعة والغابت/جلمعة الموصل في منتصف

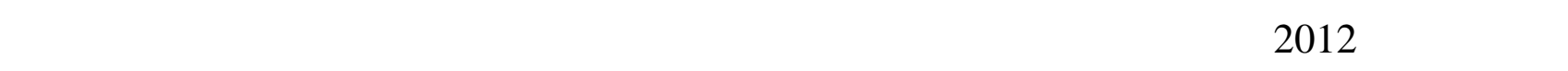

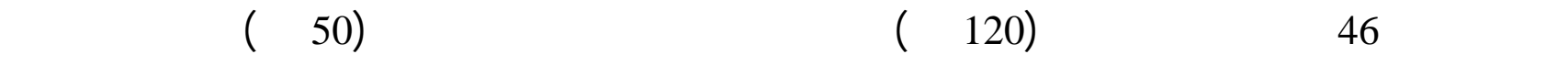

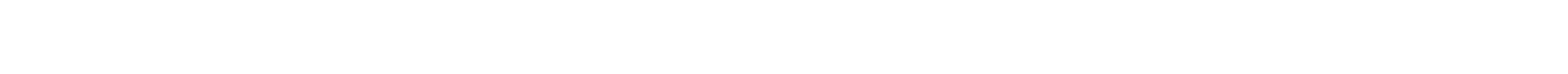

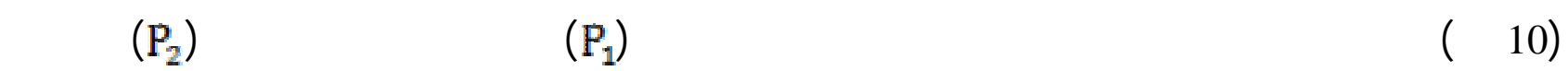
خطوط للجل الثاني (F2) وعثرة خطوط للجيل الثان

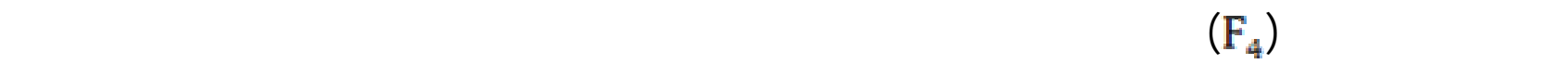

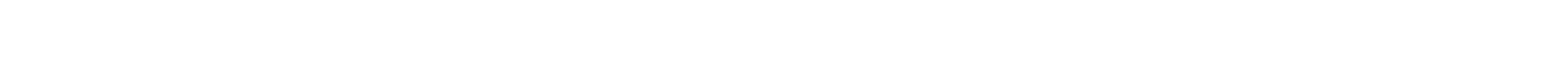

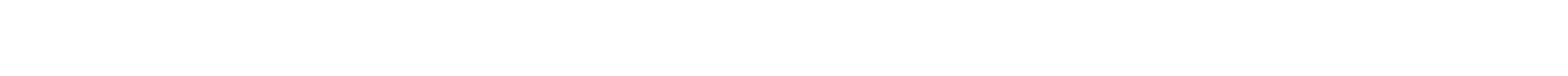

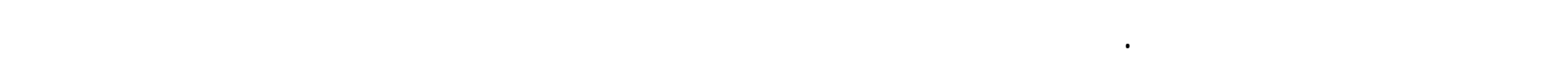
عدا النباتين الطرفيين وللصفلت الكمية: وقت طرد اللسنالب (يوم) وارتفاع النبت لهم) ونسبة الكلوروفيل الكلي في ورقة العلم وطول السنبلة (بم) وعدد عروق ورقة العلم ومسلحة ورقة العلم لسم²).

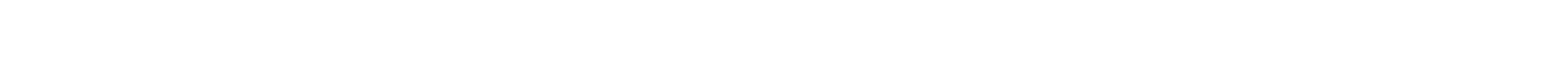

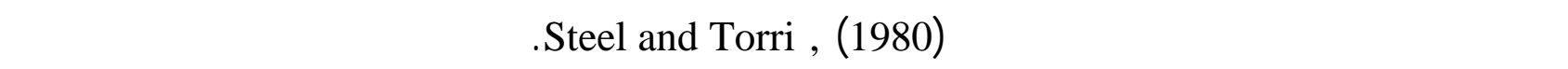
في الأجيل المستخمة للتهجينين بطريقة (1982) Mather and Jinkes, بطبق أنمونجين أنموذج المعالم الوراثية الثلاثة: ولستخدم في بداية التحليل الوراثي ويتضمن هذا الأنموذج ثلاثة معالم وراثية هي: قيمة

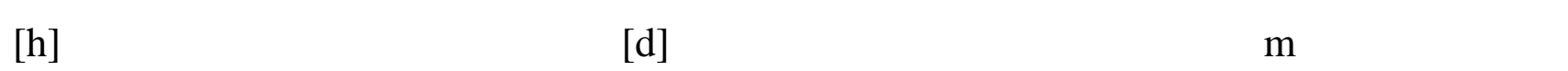

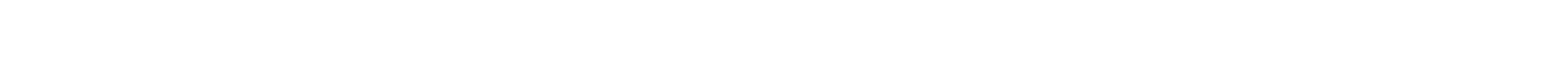

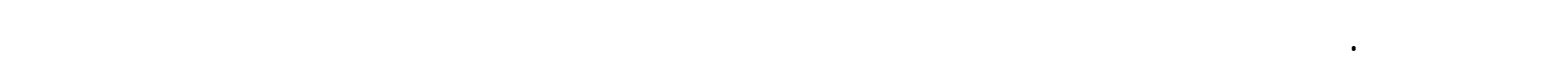

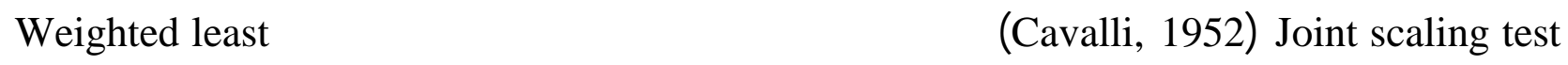
squares الأجيل المظورة وفق معادلة الانحدار الطبيعية.

$$
\widehat{\mathrm{B}}=\left(X^{\prime} W^{-1} X\right)^{-1} \cdot X^{\prime} W^{-1} \bar{y}
$$

حيث: الثلاثة ومصفوفة المعلملات ومبدول المصفوفة X ومتجه عمودي لمتوسطلت الأجيل و مصفوفة قطرية 


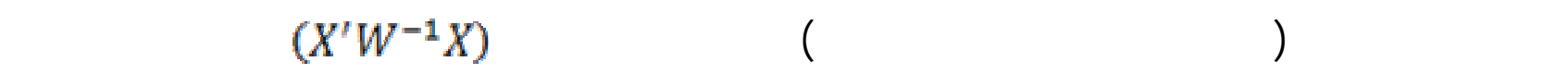

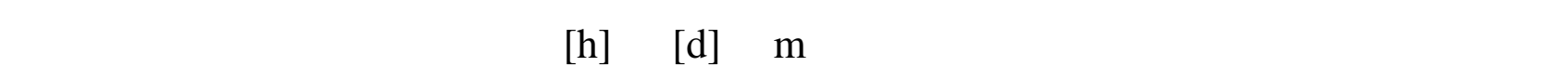
الفطرية للمصفوفة ، لختبار t حيث تحسب قيمة (t) على النحو التالي:

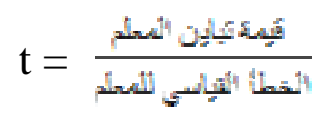

لختبرت الاختلافت بين قيم المتوطلت المسابية المظورة للأجيل ( (

$$
x_{(2)}^{2} \sum_{i=1}^{5}\left(0_{i}-E_{i}\right)^{2} \cdot(\text { Weight })_{i}
$$

تشير القيمة المعنوية لمربع كاي إلى عدم ملائمة أنموذج المعالم الوراثية الثلاثة بسببظواهر وراثية

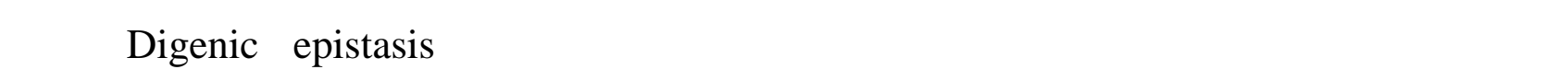

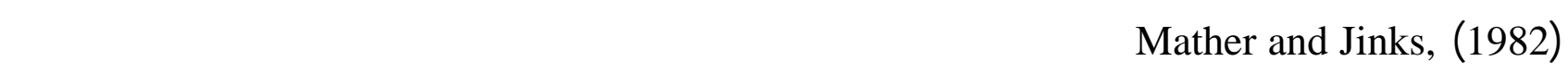

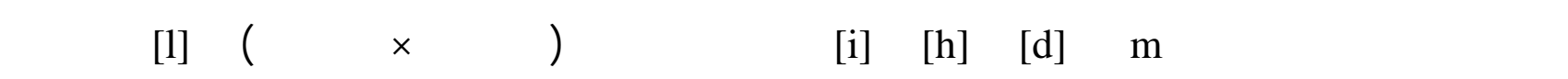

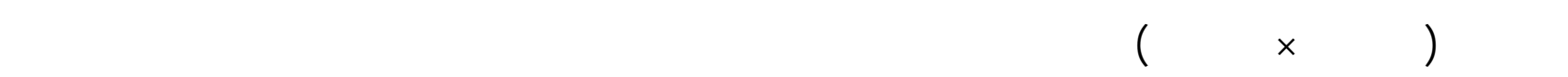
التجربة وفق المعادلات المعلة من قبل يوضف، (2009)، ولختبرت معنوية كل معلم من المعالم الوراثية

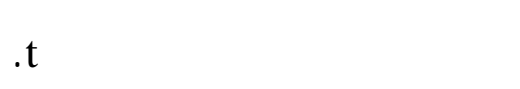

\section{النتائج و المنالثةة}

تعود الاختلافت بين قيم المتوسطت الحسابية للصفلت المدروسة في الأجيل الخمسة للتهجينين

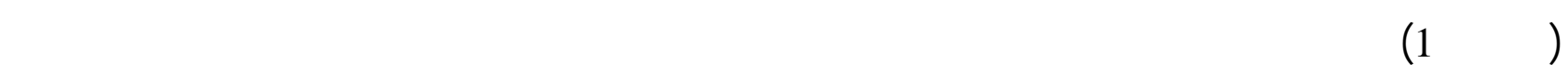

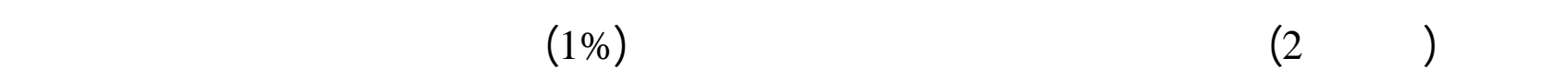
ولجمبع الصفلت المدروسة ولذا يمكن الاستمرار في التحليل الوراثي المتوسطلت تلك الأجيل.

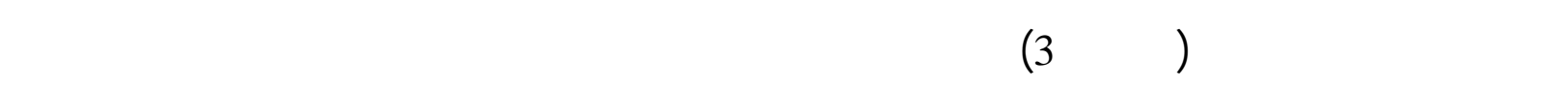

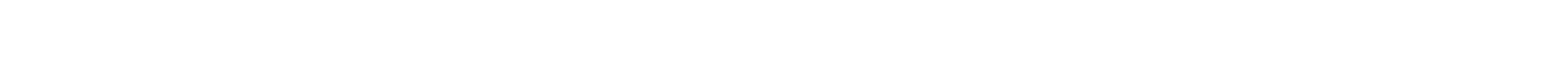

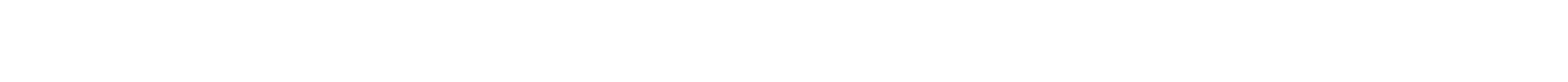

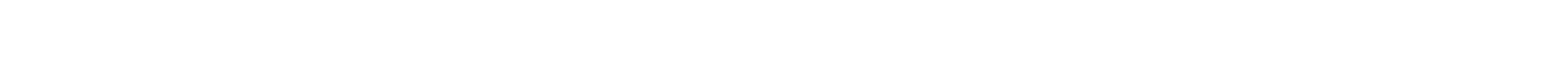

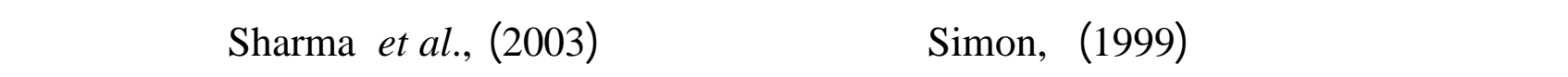
و Novoselovic et al., (2004 لارتفاع النبت والحيالي و يُونف، (2006) لطول السنبلة و و 


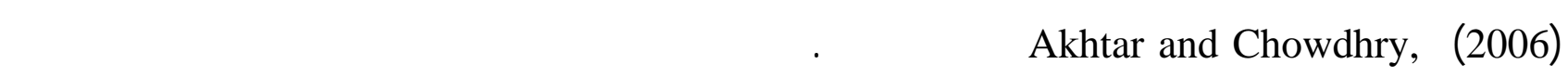
الحطة كل من (2004) Sharma and Sain لوقت طرد السنالبل وارتقاع النبلت ويوسف والحيالي، (2009) لارتقاع النبت ومسلحة ورقة العلم. ويعود عدم الفق نتائج هذه الدرلسة مع الدرلسلت الأخرى إلى الاختلاف

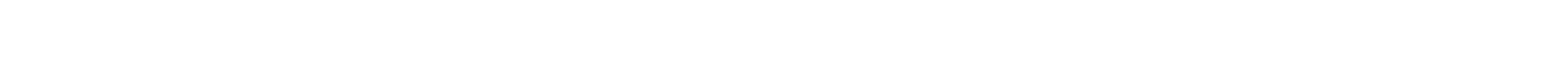

الصفلت الكمية.

يوضَح (الجدول 4) قديرات المعالم الوراثية الخمسة بموجب أنموذج الفوق ثنائي الجن وللتهجينين

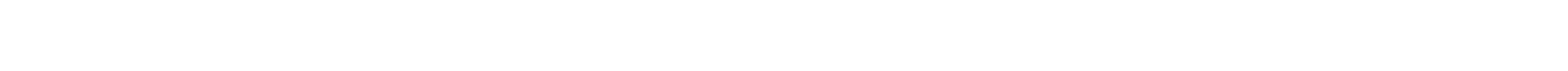

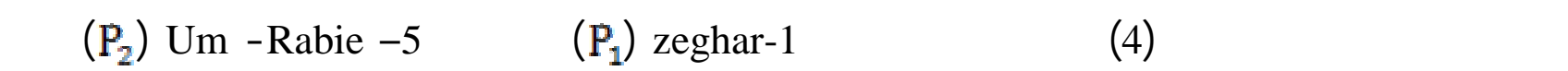
التهجين الألول، واعتبار الصف Leeds

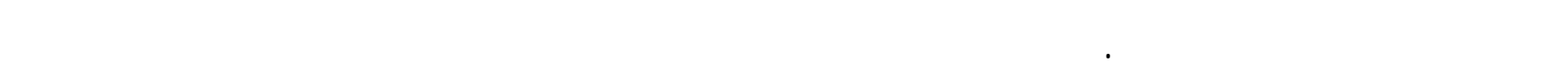

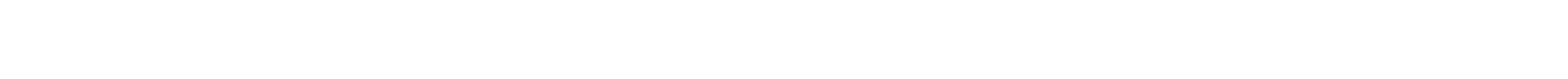

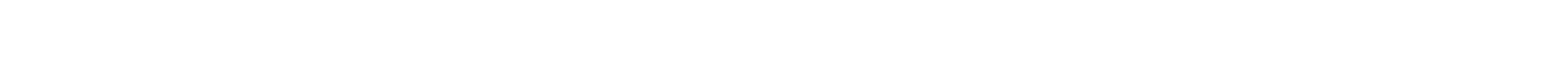

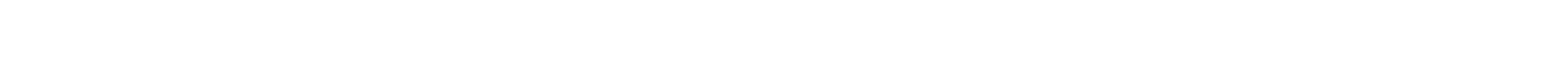

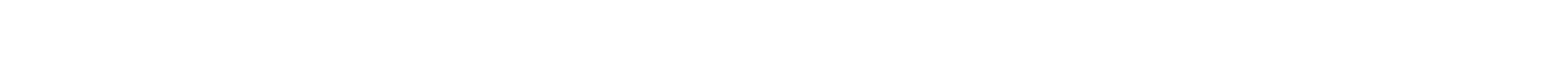

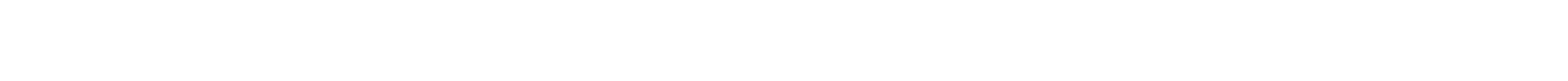

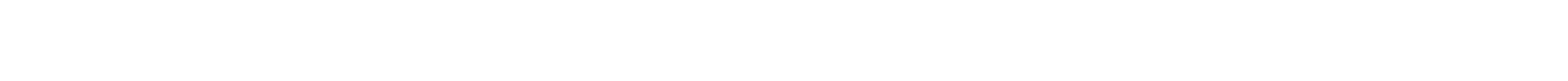

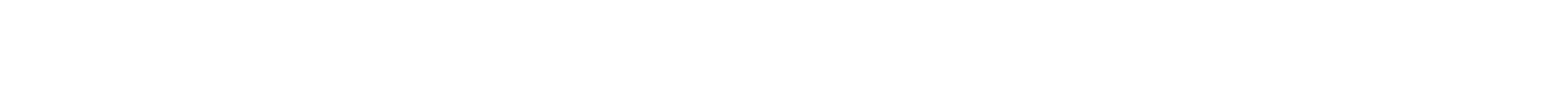

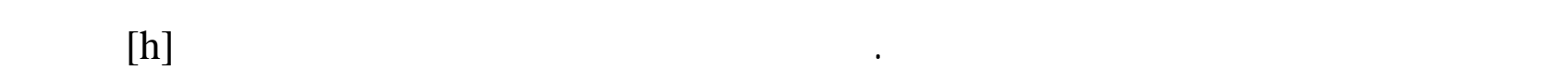

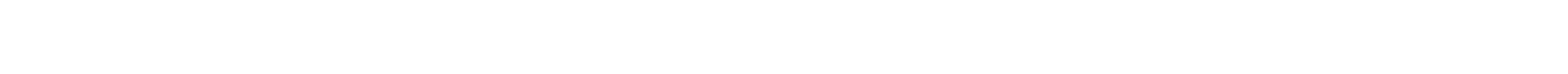

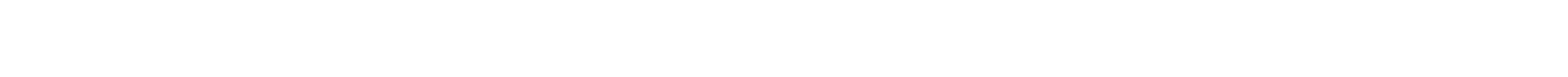

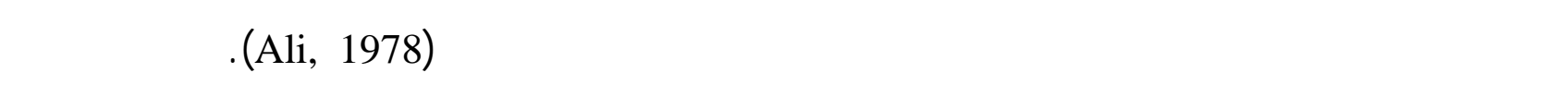

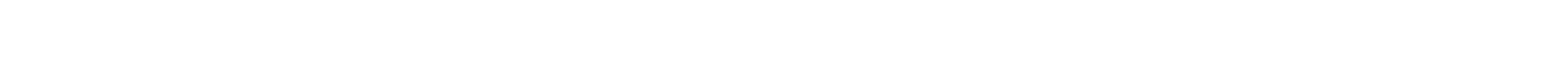

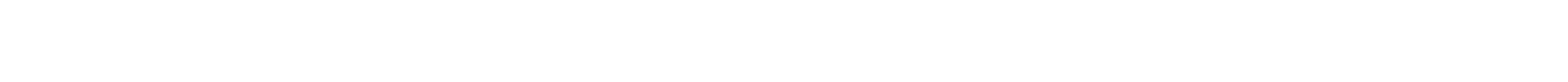
التوازن بين القيم الموجبة وللسالبة للتأثيرات للسيادية للجينات المتعددة.

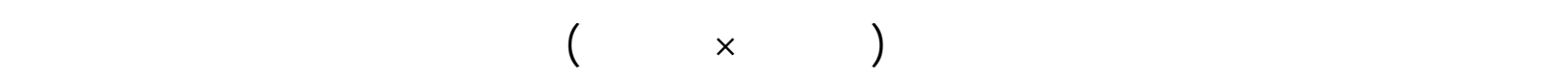
اللسنبلة وعدد عروق ورقة العلم في التهجينن ومسلحة ورقة العلم في التهجين الأول إلى إلغاء التأثيرات

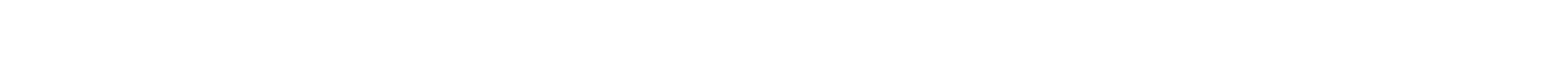

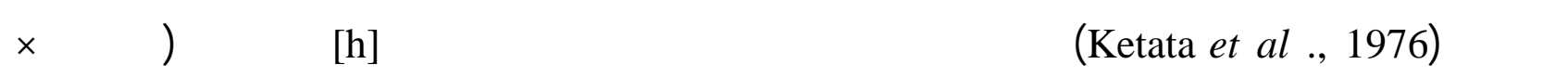

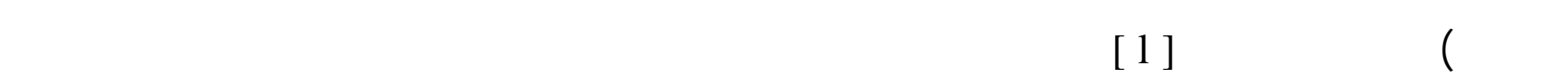




$$
\text { نجيب قاقوس يوضف وساهرمتي عبودي }
$$

لجمبع الصفات المدرووة في التهجينين، ويشير هذا التضاد إلى الفوق المضاع Duplicate Epistasis

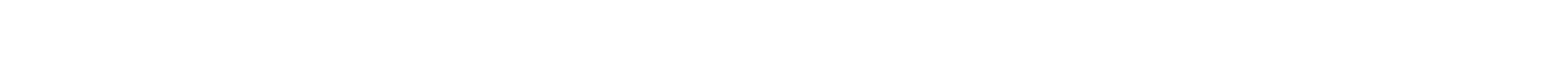
الكلوروفيل الكلي في ورقة العلم في التهجينين ومسلحة ورقة العلم في التهجين الثالي لكونه معنويا عاليا في

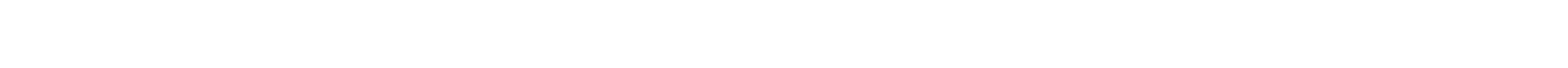

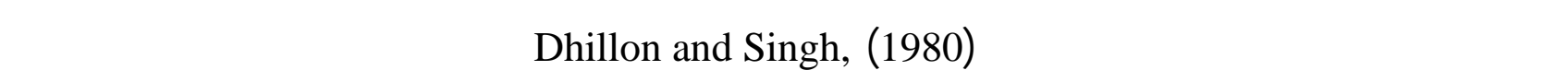
في كل من هذه التأثيرات يعتمد على مقدار التأثيرات الأخرى. تعتمد الإثارات الموجبة أو اللسالبة لقيم

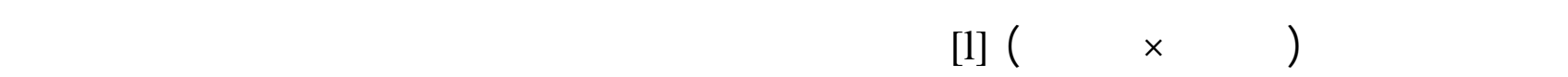
الفعل الجيني (Singh and Singh, 1980).

\section{الجرل 1: المتوطلت الهسلبية للمفل الكمية المدروسة في خطسة أجيل التهجينين الألط بين الصفين (Um- Rabie-5 × Azeghar-1 ) والثلي بين الصفين (Waha X Leeds) في الحظلة}

\begin{tabular}{|c|c|c|c|c|c|c|c|}
\hline النفاع & طلط السنبلة & مسلة ورقة & ورقة اللهرة & الكلي فبة الكلورولل & قالمنطلالل & التهجني & الجي لل \\
\hline $\begin{array}{l}82.783 \\
\pm 0.235\end{array}$ & $\begin{array}{c}9.917 \\
\pm 0.193\end{array}$ & $\begin{array}{l}37.996 \\
\pm 0.334\end{array}$ & $\begin{array}{r}13.533 \\
\pm 0.448\end{array}$ & $\begin{array}{l}47.801 \\
\pm 0.266\end{array}$ & $\begin{array}{r}33.500 \\
\pm 0.292\end{array}$ & الأول & \multirow{2}{*}{$P_{1}$} \\
\hline $\begin{array}{l}69.683 \\
\pm 0.197\end{array}$ & $\begin{array}{c}8.200 \\
\pm 0.193\end{array}$ & $\begin{array}{l}30.570 \\
\pm 0.318\end{array}$ & $\begin{array}{c}8.033 \\
\pm 0.432\end{array}$ & $\begin{array}{l}42.176 \\
\pm 0.196\end{array}$ & $\begin{array}{l}35.383 \\
\pm 0.238\end{array}$ & الثاني & \\
\hline $\begin{array}{l}83.283 \\
\pm 0.283\end{array}$ & $\begin{array}{c}8.653 \\
\pm 0.222\end{array}$ & $\begin{array}{r}43.543 \\
\pm 0.393\end{array}$ & $\begin{array}{r}14.733 \\
\pm 0.502\end{array}$ & $\begin{array}{l}48.707 \\
\pm 0.265\end{array}$ & $\begin{array}{l}30.366 \\
\pm 0.228\end{array}$ & الأول & \multirow{2}{*}{$P_{2}$} \\
\hline $\begin{array}{l}68.966 \\
\pm 0.223\end{array}$ & $\begin{array}{c}9.700 \\
\pm 0.241\end{array}$ & $\begin{array}{l}35.453 \\
\pm 0.361\end{array}$ & $\begin{array}{c}9.166 \\
\pm 0.380\end{array}$ & $\begin{array}{l}43.976 \\
\pm 0.240\end{array}$ & $\begin{array}{l}36.050 \\
\pm 0.215\end{array}$ & الثالي & \\
\hline $\begin{array}{l}84.730 \\
\pm 0.327\end{array}$ & $\begin{array}{c}8.960 \\
\pm 0.260\end{array}$ & $\begin{array}{l}43.733 \\
\pm 0.405\end{array}$ & $\begin{array}{l}14.840 \\
\pm 0.569\end{array}$ & $\begin{array}{l}49.473 \\
\pm 0.325\end{array}$ & $\begin{array}{l}33.330 \\
\pm 0.316\end{array}$ & الأول & \multirow{2}{*}{$F_{2}$} \\
\hline $\begin{array}{l}69.230 \\
\pm 0.296\end{array}$ & $\begin{array}{c}8.360 \\
\pm 0.250\end{array}$ & $\begin{array}{l}35.395 \\
\pm 0.362\end{array}$ & $\begin{array}{c}9.480 \\
\pm 0.451\end{array}$ & $\begin{array}{l}45.227 \\
\pm 0.299\end{array}$ & $\begin{array}{l}36.050 \\
\pm 0.270\end{array}$ & الثالي & \\
\hline $\begin{array}{l}85.750 \\
\pm 0.321\end{array}$ & $\begin{array}{l}10.160 \\
\pm 0.238\end{array}$ & $\begin{array}{l}43.427 \\
\pm 0.349\end{array}$ & $\begin{array}{l}15.000 \\
\pm 0.518\end{array}$ & $\begin{array}{l}49.915 \\
\pm 0.295\end{array}$ & $\begin{array}{l}33.445 \\
\pm 0.265\end{array}$ & الأول & \multirow{2}{*}{$F_{3}$} \\
\hline $\begin{array}{l}73.165 \\
\pm 0.319\end{array}$ & $\begin{array}{c}9.620 \\
\pm 0.229\end{array}$ & $\begin{array}{l}34.080 \\
\pm 0.324\end{array}$ & $\begin{array}{l}10.605 \\
\pm 0.435\end{array}$ & $\begin{array}{l}46.673 \\
\pm 0.301\end{array}$ & $\begin{array}{l}36.470 \\
\pm 0.250\end{array}$ & الثاني & \\
\hline $\begin{array}{r}89.984 \\
\pm 0.264 \\
\end{array}$ & $\begin{array}{r}11.060 \\
\pm 0.183 \\
\end{array}$ & $\begin{array}{r}44.089 \\
\pm 0.278 \\
\end{array}$ & $\begin{array}{r}16.560 \\
\pm 0.388 \\
\end{array}$ & $\begin{array}{l}52.195 \\
\pm 0.237\end{array}$ & $\begin{array}{l}33.578 \\
\pm 0.199\end{array}$ & الأول & \multirow{2}{*}{$F_{4}$} \\
\hline $\begin{array}{l}79.978 \\
\pm 0.271\end{array}$ & $\begin{array}{l}10.576 \\
\pm 0.189\end{array}$ & $\begin{array}{l}35.188 \\
\pm 0.266\end{array}$ & $\begin{array}{l}12.352 \\
\pm 0.337\end{array}$ & $\begin{array}{l}50.191 \\
\pm 0.240\end{array}$ & $\begin{array}{l}36.940 \\
\pm 0.189\end{array}$ & الثاني & \\
\hline
\end{tabular}




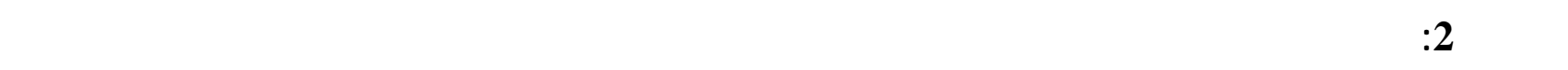
(Um- Rabie-5 XAzeghar-1)

\begin{tabular}{|c|c|c|c|c|c|c|c|c|}
\hline \multicolumn{6}{|c|}{ متوس ط الم ـرعs ـ _ ــات } & \multirow[b]{2}{*}{ النهجين } & \multirow[b]{2}{*}{ الحربة } & \multirow[b]{2}{*}{ التبلان } \\
\hline $\begin{array}{c}\text { ارفاع النبلت (سم ) } \\
\text { (سماع }\end{array}$ & طل السنبلة & 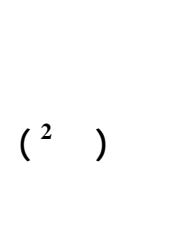 & عرقة الهلم & الكلورولى الكالية & 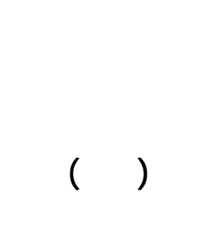 & & & \\
\hline 153.538 & 17.578 & 2.281 & 44.586 & 5.229 & 238.522 & الأول & \multirow{2}{*}{3} & \multirow[b]{2}{*}{ المكررت } \\
\hline 7.223 & 76.664 & 361.412 & 123.333 & 50.301 & 7.508 & الثاني & & \\
\hline$* * 1714.941$ & $* * 167.782$ & $* * 499.214$ & **234.518 & **525.438 & **140.387 & الأول & \multirow{2}{*}{4} & \multirow{2}{*}{ ل الأجيل } \\
\hline$* * 4865.412$ & $* * 175.429$ & $* * 325.408$ & $* * 564.178$ & $* * 542.779$ & $* * 49.520$ & الثالي & & \\
\hline 100.130 & 2.922 & 9.723 & 3.500 & 4.825 & 32.374 & الأول & \multirow{2}{*}{12} & \multirow{2}{*}{ الجربيأ } \\
\hline 21.299 & 4.208 & 4.882 & 221.445 & 21.97 & 4.702 & الثاني & & \\
\hline 65.525 & 5.690 & 23.757 & 17.367 & 21.211 & 13.267 & الأول & \multirow{2}{*}{900} & \multirow{2}{*}{ الهيف } \\
\hline 97.550 & 5.013 & 25.380 & 8.427 & 15.725 & 5.903 & الثاني & & \\
\hline
\end{tabular}

1\% معنوي عند مستوى لحتمل ** *

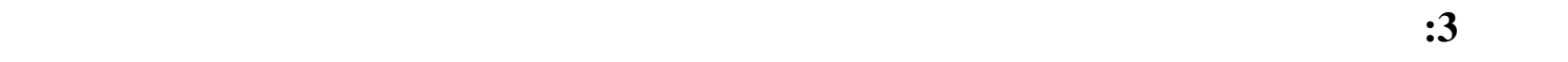

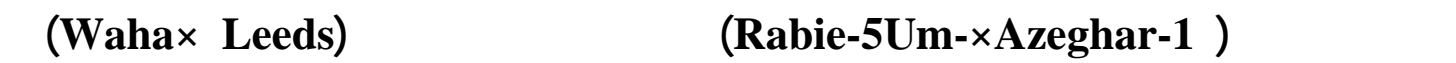

الهظلة النشنة

\begin{tabular}{|c|c|c|c|c|c|c|c|}
\hline 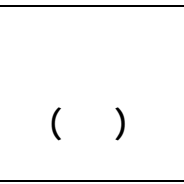 & طلط السنبلة & مساحة ورقة العلم & عدد عرق ورقة & الكليف فسبة الكلورفل العلم & 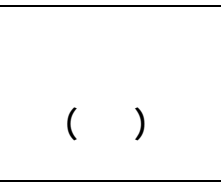 & التهجين & المالمعالم \\
\hline $\begin{array}{c}* * 84.776 \\
\pm 0.162 \\
\end{array}$ & $\begin{array}{c}* * 9.921 \\
\pm 0.126 \\
\end{array}$ & $\begin{array}{c}* * 68.580 \\
\pm 0.213 \\
\end{array}$ & $\begin{array}{c}* * 14.926 \\
\pm 0.284 \\
\end{array}$ & $\begin{array}{c}* * 49.308 \\
\pm 0.154 \\
\end{array}$ & $\begin{array}{c}* * 32.467 \\
\pm 0.154 \\
\end{array}$ & الأول & \multirow[b]{2}{*}{$\mathrm{m}$} \\
\hline $\begin{array}{c}* * 71.365 \\
\pm 0.137\end{array}$ & $\begin{array}{c}* * 9.586 \\
\pm 0.131\end{array}$ & $\begin{array}{c}* * 33.560 \\
\pm 0.199\end{array}$ & $\begin{array}{c}* * 9.904 \\
\pm 0.242\end{array}$ & $\begin{array}{c}* * 44.674 \\
\pm 0.139\end{array}$ & $\begin{array}{c}* * 36.135 \\
\pm 0.136\end{array}$ & الثاني & \\
\hline $\begin{array}{c}* *-0.572 \\
\pm 0.183\end{array}$ & $\begin{array}{c}* * 0.542 \\
\pm 0.147\end{array}$ & $\begin{array}{c}* *_{-} 8.297 \\
\pm 0.257\end{array}$ & $\begin{array}{l}*_{-} 0.690 \\
\pm 0.336\end{array}$ & $\begin{array}{l}-0.310 \\
\pm 0.176\end{array}$ & $\begin{array}{c}1.696 \\
\pm 0.184\end{array}$ & الأول & \multirow[b]{2}{*}{ [d] } \\
\hline $\begin{array}{l}0.115 \\
0.149 \\
\end{array}$ & $\begin{array}{c}* *_{-} 0.889 \\
\pm 0.153\end{array}$ & $\begin{array}{c}* *-2.510 \\
\pm 0.240\end{array}$ & $\begin{array}{l}-0.399 \\
\pm 0.287\end{array}$ & $\begin{array}{c}* *-1.213 \\
\pm 0.154\end{array}$ & $\begin{array}{l}*_{-}-0.291 \\
\pm 0.160\end{array}$ & الثاني & \\
\hline $\begin{array}{c}* * 3.642 \\
\pm 0.724\end{array}$ & $\begin{array}{l}-0.339 \\
\pm 0.573 \\
\end{array}$ & $\begin{array}{c}* *_{-}-48.577 \\
\pm 0.924\end{array}$ & $\begin{array}{c}1.167 \\
\pm 1.272 \\
\end{array}$ & $\begin{array}{c}* * 2.666 \\
\pm 0.707\end{array}$ & $\begin{array}{c}* * 2.981 \\
\pm 0.700\end{array}$ & الأول & \multirow[b]{2}{*}{ [h] } \\
\hline $\begin{array}{c}* * 1.860 \\
\pm 0.636\end{array}$ & $\begin{array}{l}-1.100 \\
\pm 0.566 \\
\end{array}$ & $\begin{array}{c}* * 4.083 \\
\pm 0.839 \\
\end{array}$ & $\begin{array}{c}1.523 \\
\pm 1.037 \\
\end{array}$ & $\begin{array}{c}* * 5.489 \\
\pm 0.645 \\
\end{array}$ & $\begin{array}{c}0.734 \\
\pm 0.605 \\
\end{array}$ & الثاني & \\
\hline **190.634 & $* * 66.086$ & $* * 49854.7$ & **21.802 & $* * 161.644$ & $* * 26.691$ & الأول & \multirow[t]{2}{*}{$X^{2}(2)$} \\
\hline **1242.532 & **89.176 & $* * 25.591$ & $* * 72.807$ & **572.649 & $* * 24.231$ & الثاني & \\
\hline
\end{tabular}

ت تشير إلى قيمة متووط الأبوين والتأثيرات الإضفية وللسيادية للجينت المتعدة على التوالي. 
نجيب قاقوس يوسف وساهر متي عبودي

الجرط 4: قفيرات المعالم الوراثية الخصة للصفل الكمية المدروسة في التهجينين الأل ببن ال صفين

والثلي بين الصفين (Um- Rabie-5 X Azeghar-1 )

الهشنة

\begin{tabular}{|c|c|c|c|c|c|c|c|}
\hline 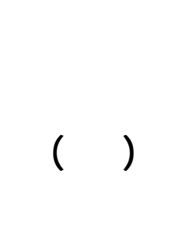 & طل للسنبلة & مساحة ورقة & عدق عرق العلم & الكالي فلورفل ورقة & (لسنالل & التهجين & الوراثية \\
\hline $\begin{array}{c}* * 96.701 \\
\pm 0.958\end{array}$ & $\begin{array}{c}* * 12.160 \\
\pm 0.686\end{array}$ & $\begin{array}{c}* * 45.294 \\
\pm 1.028\end{array}$ & $\begin{array}{c}* * 19.107 \\
\pm 1.476 \\
\end{array}$ & $\begin{array}{c}* * 55.849 \\
\pm 0.871 \\
\end{array}$ & $\begin{array}{c}* * 33.761 \\
\pm 0.758\end{array}$ & الأول & \multirow[b]{2}{*}{$\mathrm{m}$} \\
\hline $\begin{array}{c}* * 90.021 \\
\pm 0.969\end{array}$ & $\begin{array}{c}* * 11.749 \\
\pm 0.686\end{array}$ & $\begin{array}{c}* * 37.473 \\
\pm 0.968\end{array}$ & $\begin{array}{c}* * 14.889 \\
\pm 1.260\end{array}$ & $\begin{array}{c}* * 55.574 \\
\pm 0.883\end{array}$ & $\begin{array}{c}* * 37.583 \\
\pm 0.716\end{array}$ & الثالي & \\
\hline $\begin{array}{l}-0.250 \\
\pm 0.184\end{array}$ & $\begin{array}{c}* * 0.632 \\
\pm 0.147\end{array}$ & $\begin{array}{c}* *-2.773 \\
\pm 0.258\end{array}$ & $\begin{array}{l}-0.600 \\
\pm 0.337\end{array}$ & $\begin{array}{l}*_{-} 0.453 \\
\pm 0.188\end{array}$ & $\begin{array}{c}* * 1.567 \\
\pm 0.185\end{array}$ & الأول & \multirow[b]{2}{*}{ [d] } \\
\hline $\begin{array}{l}* 0.359 \\
\pm 0.149\end{array}$ & $\begin{array}{c}* *-0.750 \\
\pm 0.154\end{array}$ & $\begin{array}{c}* *-2.442 \\
\pm 0.240\end{array}$ & $\begin{array}{l}*_{-} 0.567 \\
\pm 0.288\end{array}$ & $\begin{array}{c}* *_{-} 0.900 \\
\pm 0.155\end{array}$ & $\begin{array}{c}{ }_{-}-0.333 \\
\pm 0.161\end{array}$ & الثالني & \\
\hline $\begin{array}{c}* *-63.000 \\
\pm 7.782 \\
\end{array}$ & $\begin{array}{l}-9.600 \\
\pm 5.679\end{array}$ & $\begin{array}{l}-11.816 \\
\pm 8.445\end{array}$ & $\begin{array}{c}*-24.320 \\
\pm 12.286\end{array}$ & $\begin{array}{c}* *-34.725 \\
\pm 7.135 \\
\end{array}$ & $\begin{array}{l}-1.668 \\
\pm 6.309\end{array}$ & الأول & \multirow[b]{2}{*}[\mathrm{h}]{} \\
\hline $\begin{array}{c}* *-93.268 \\
\pm 7.804\end{array}$ & $\begin{array}{l}-10.256 \\
\pm 5.582\end{array}$ & $\begin{array}{c}* *-23.588 \\
\pm 7.888\end{array}$ & $\begin{array}{c}* *-23.452 \\
\pm 10.389\end{array}$ & $\begin{array}{c}* *-50.517 \\
\pm 7.231\end{array}$ & $\begin{array}{l}-5.840 \\
\pm 5.947\end{array}$ & الثالي & \\
\hline $\begin{array}{c}* *_{-}-13.668 \\
\pm 0.975\end{array}$ & $\begin{array}{c}* *-2.875 \\
\pm 0.702\end{array}$ & $\begin{array}{c}* *_{-}-4.5243 \\
\pm 1.060\end{array}$ & $\begin{array}{c}* *_{-} 4.973 \\
\pm 1.514\end{array}$ & $\begin{array}{c}* *-7.596 \\
\pm 0.891\end{array}$ & $\begin{array}{c}*_{-}-1.828 \\
\pm 0.780\end{array}$ & الأول & \multirow[b]{2}{*}{ [i] } \\
\hline $\begin{array}{c}* *-20.697 \\
\pm 0.980\end{array}$ & $\begin{array}{c}* *-2.799 \\
\pm 0.703\end{array}$ & $\begin{array}{c}* *-4.461 \\
\pm 0.998\end{array}$ & $\begin{array}{c}* *-6.289 \\
\pm 1.292\end{array}$ & $\begin{array}{c}* *-12.498 \\
\pm 0.896\end{array}$ & $\begin{array}{l}*-1.867 \\
\pm 0.734\end{array}$ & الثاني & \\
\hline $\begin{array}{c}* * 79.445 \\
\pm 12.204\end{array}$ & $\begin{array}{c}6.400 \\
\pm 8.990\end{array}$ & $\begin{array}{c}17.386 \\
\pm 13.382\end{array}$ & $\begin{array}{c}31.573 \\
\pm 19.491\end{array}$ & $\begin{array}{c}* * 43.942 \\
\pm 11.260\end{array}$ & $\begin{array}{c}1.611 \\
\pm 10.059\end{array}$ & الأول & \multirow[b]{2}{*}{ [1] } \\
\hline $\begin{array}{c}* * 103.371 \\
\pm 12.148\end{array}$ & $\begin{array}{c}6.955 \\
\pm 8.784\end{array}$ & $\begin{array}{c}* * 37.664 \\
\pm 12.437\end{array}$ & $\begin{array}{c}25.269 \\
\pm 16.382\end{array}$ & $\begin{array}{c}* * 59.644 \\
\pm 11.353\end{array}$ & $\begin{array}{c}5.547 \\
\pm 9.418\end{array}$ & الثاني & \\
\hline
\end{tabular}

* و * معنوية عند مستوى لحتمل 5\% م و 1\% على التوالمي.

تشير القيم المعنوية العالية للتأثيرات القوقية للجينت المتعدة (للسيادية × للسيادية) مقارينة بالقيم الوطئة للتأثيرات الفوقية للجينت المتعدة (الإضفية × الإضفية) لكل من ارقفاع النبت ومحتوى الكلوروفل وعدد عروق ورقة العلم في التهجينين، مسلحة ورقة العلم في التهجن الثاني إله إن الجينت المتعدة التي تعين تلك الصفلت في التهجينين هي منتشرة كليا أو غير مرتبطة جزئيا (Pantanaik and Murty , 1978). 
يمكن لستغلل الانتخلب المبلثر Direct selection في الأجيل الانعزالية المستخدة في هذه الدرلسة

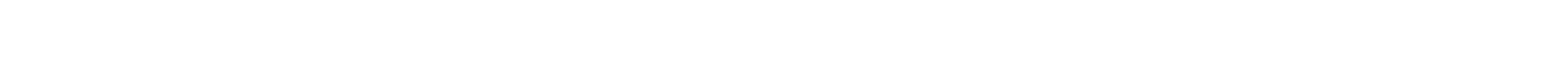

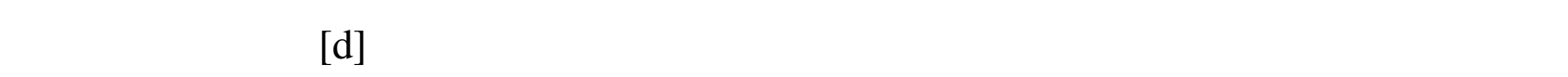

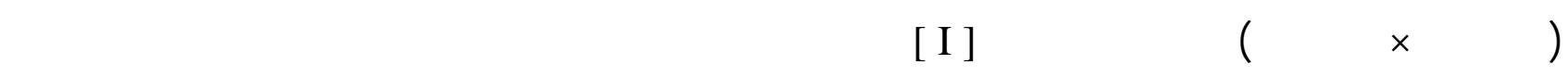

غيرمعنوي.

ويعد الانتخلب المتكرر Recurrent Selection أفضل طريقة لزياة تكرار الجينات المرغوبة لتعسين الصفلت الأخرى في التهجينين، وذك للدور المعنوي الذي هُقته التأثيرات الإضفافية وغير الإضفافية للجينات

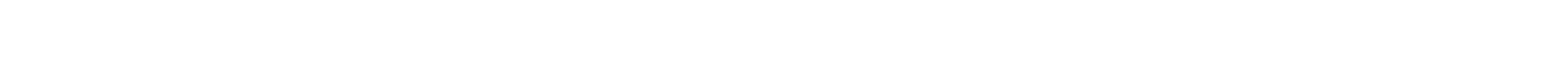

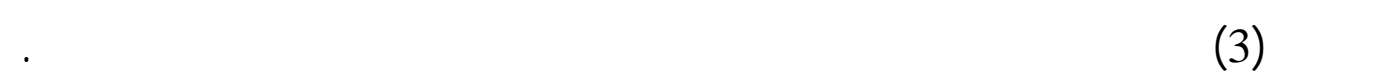

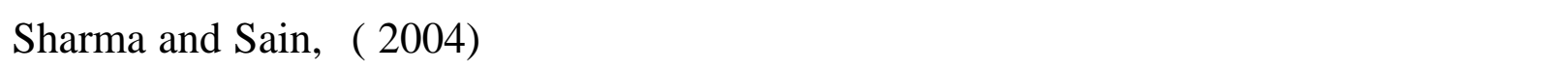
لارتقاع النبلت ومسلحة ورقة العلم و Munir et al., لطول (2007) للسنبلة وعدد عروق ورقة العلم و Fethi et al., لارفقاع النبلت وطول اللسنبلة ومسلحة

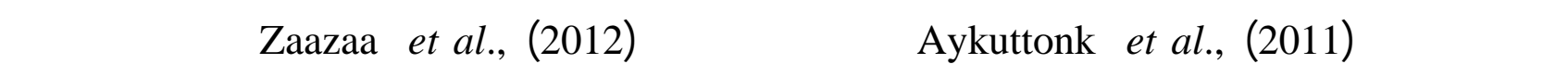
البدراني ولخرون (2012) لارفقاع النبلت ووقت وطرد للسنالب.

\section{المصادر المربية}

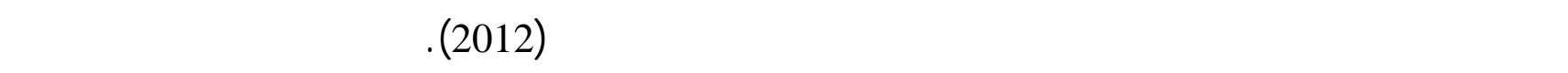

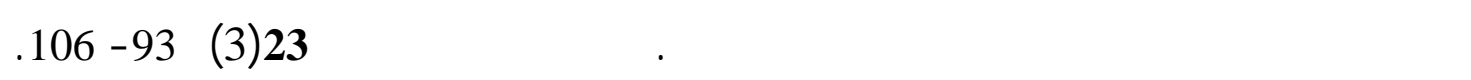

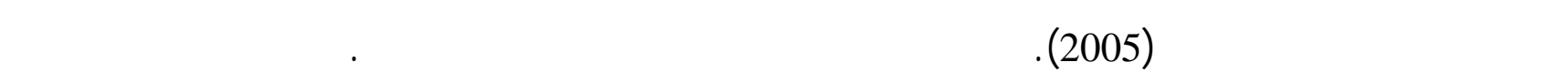
علوم الحية، كلية العلو، جلمعة الموصل.

يوسف، نجيب قاقوس؛ الحيالي، منل عبد المطلب (2006). التحليل الوراثي لمتوسطت الأجيل المبكرة في

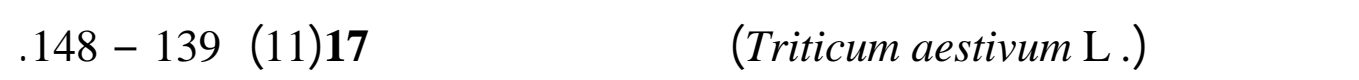

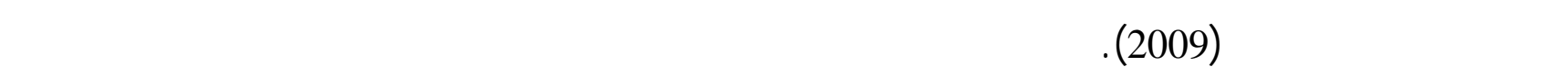
الصفات في عدة لجيل لتهجينين من الثعير.مجلة زراعة الرافين، 37(2)، 2004 - 217.

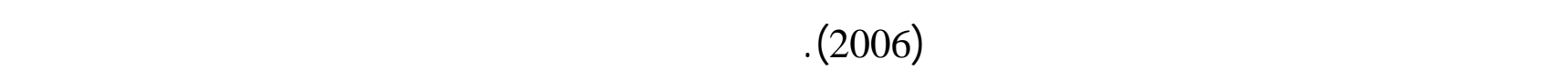

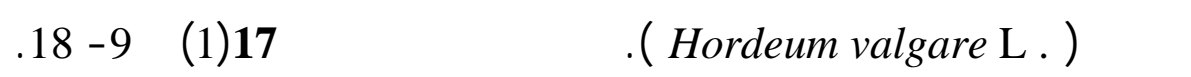
الحمدالي، غادة عبد الة؛ يوضف، نجيب قاقوس (2006). نقدير الفعل الجيني والتوريث لبعض الصفلت في الحطة الغشنة، مجلة زراعة الرافين، 43(3)، 108 - 118. 


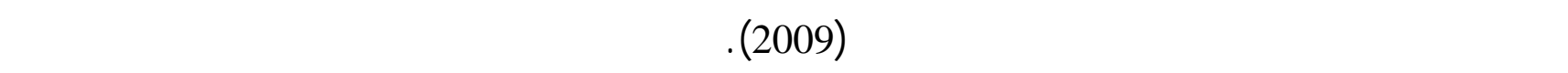

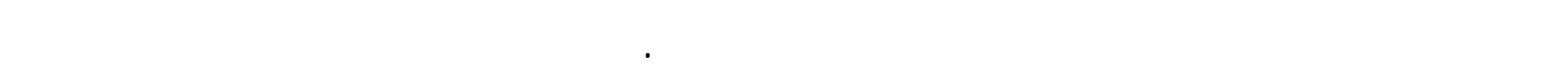
كلية العلوم، جلمعة الموصل، 216 - 222.

\section{المصار الأجنبية}

Akhtar, N. ; Chowdhry, M.A. (2006). Genetic analysis of yield and some other quantitative traits in bread wheat. Int. J. Agri. Biol., 8(4),523-527.

Ali, M.I. (1978). Estimates of genetic effects of eight agronomic characters and their interrelationships in a spring wheat. Alex J. Agric. Res., 26(3), 599-607.

Aykuttonk, F.; Ilker, E.; Tosun, M. (2011). Quantitative inheritance of some wheat agronomic traits. Bulg. Agric. Sci., 17, 783-788.

Cavalli, L.L. (1952). "An Analysis of Linkage in Qauantitative Inheritance". In E.C. Reeve and C.H. Waddington, eds, Quantitative Inheritance. Her Majestrys Stationery Office, London, UK.

Dhillon, S.S.; Singh, T.H. (1980). Genetic control of some quantitative characters in upland cotton.( Gossypium hirsutum L. ). J. Agric. Sci. Camb., 94, 530-543.

Fethi, B. ; Mohamed, E.M. (2010). Epistasis and genotype-by-environment interaction of grain yield related traits in durum wheat. J. Plant Breed. Crop Sci., 2(2), 24-29.

Gamble, E.E. (1962). Gene effect in corn, (Zea mays L.). I. separation and relative importance of gene effects for yield. Can. J. Plant Sci., 42, 339-348.

Hayman, B.M. (1958). The separation of epistatic from additive and dominance variation in generation mean. Heredity., 12, 371-390.

Kasim, M.H.; Yousif, N.K. (1990). Estimating gene effect by analysis self-fertilized generation mean in barley. Mesopotamia J. Agric., 22(3), 15-22.

Ketata, H.; Edwards, L.H.; Smith, E.L. (1976). Inheritance of eight agronomic characters in a winter wheat cross. Crop. Sci., 16,19-22.

Khattab, S.A.M.; Esmail, R.M.; Alnasary, A.E-R.M.F. (2010). Genetical analysis of some quantitative traits in bread wheat ( Triticum aestivum L.). New York. Sci. J., 3(11), 152-157.

Mather, K. (1949). Biometrical Genetics. $l^{\text {st }}$ edition, Dover publication, Inc., London.

Mather, K.; Jinks, J.L. (1982). Biometrical Genetics. $3^{\text {rd }}$ edition, Chapman and Hall, ltd., London.

Munir, M.; Chowdhry, M.A.; Ahsan, M. (2007). Generation means studies in bread wheat under drought condition. Inter. J. Agric. Biol., 9(2), 282-286.

Novoselovic, D.; Baric, M.; Drezener, G.; Gunjaca, J.; Lalic, A. (2004). Quantitative inheritance of some wheat plant traits. Genetics and Molecular Biology., 27(1), 9298.

Pantanaik, M.; Murty, B.R. ( 1978 ) . Gene action and heterosis in brown sarson. Indian. J. Genet. PI. Breed. 38(1), 119-125.

Simon, M.R. (1999). Inheritance of flag-leaf angle, flag-leaf area and flag-leaf area duration in four wheat crosses. Theor Appl. Genet., 98, 310-314. 
Singh, S.; Singh, R.B. (1980). A-study of gene effects in three wheat crosses. J. Agric. Sci. Camb., 91, 9-12.

Sharma, S.N.; Sain, R.S.; Sharma, R.K. (2003). Genetics of spike length in durum wheat. Euphytica., 130, 155-161.

Sharma, S.N.; Sain, R.S. (2004). Inheritance of days to heading, days to maturity, plant height and grain yield in an intervariental cross of durum wheat. J. Breeding and Genetics., 36(2), 73-82.

Steel, R.G.B.; Torrie, J.H. (1980). Principle and procedures of statistics : abiometrical approach. McGraw Hill Books Co. New York, USA.

Zaazaa, E.L.; Hager, M.A.; EL-Hashash, E.F. (2012). Genetical analysis of some quantitative traits in wheat using six parameters genetic model. American-Eurasian J. Agric and Environ. Sci., 12(4), 456-462. 